\title{
Clinico-hematological profile and morphological features on bone marrow aspirate in visceral leishmaniasis from non-endemic region
}

\author{
Gupta A.K. ${ }^{1}$, Chandra H. ${ }^{2}$, Bharati V. ${ }^{3}$, Singh N. ${ }^{4}$, Kishore S. ${ }^{5}$ \\ ${ }^{1}$ Dr. Arvind Kumar Gupta, Assistant Professor, ${ }^{2}$ Dr. Harish Chandra, Additional Professor, ${ }^{3}$ Dr. Vandana Bharati, \\ Resident, ${ }^{4}$ Dr. Neha Singh, Associate Professor, ${ }^{5}$ Dr. Sanjeev Kishore, Professor, Department of Pathology and Lab \\ Medicine, All authors are affiliated with All India Institute of Medical Sciences, Rishikesh, Uttarakhand, India.
}

Corresponding Author: Dr. Harish Chandra, Additional Professor, Department of Pathology and Lab Medicine, All India Institute of Medical Sciences, Rishikesh, Uttarakhand, India. E-mail: drharishbudakoti31@yahoo.co.in

\begin{abstract}
Introduction- Leishmaniasis which is caused by Leishmania donovani is considered to be a disease of lowland as climate and geographical factors play an important role in its distribution. However, recently few studies have reported leishmaniasis at higher altitude of Himalayan and sub Himalayan region of India. The present study was therefore conducted to study the clinico- hematological profile and the various morphological features on bone marrow examination associated with leishmaniasis in the non-endemic sub Himalayan region of India. Material and MethodsThe study included all the cases of leishmaniasis which were diagnosed on bone marrow examination over a period of one and half year in a medical institute situated in Uttarakhand state of India. Results- The study included total 10 cases of visceral leishmaniasis diagnosed on bone marrow aspirate with fever (10 cases) and hepato-splenomegaly ( 7 cases) being most common clinical presentation and pancytopenia (9 cases) being the most common haematological finding. Bone marrow aspirate cytology showed that plasmacytosis ( 9 cases) and hemophagocytosis (8 cases) were the most common features. One case was clinically suspected as acute leukemia which turned out to be a case of leishmaniasis on bone marrow examination. Conclusion- Leishmaniasis is emerging as an important major parasitic infection in the nonendemic Himalayan and sub Himalayan region of India. Fever, splenomegaly and pancytopenia are important clinical and peripheral blood examination features associated with this disease. Hemophagocytosis and plasmacytosis on bone marrow examination should prompt for vigilant search for LD bodies in bone marrow to avoid missing of diagnosis even in unsuspected cases.
\end{abstract}

Keywords: Leishmaniasis; Bone marrow aspirate; Pancytopenia; Plasmacytosis

\section{Introduction}

Visceral Leishmaniasis (VL) is parasitic disease caused by Leishmania donovani (LD) with Phlebotomus sand fly being the vector of the disease. It results from the infection of macrophages in the reticulo-endothelial system associated with immune-inflammatory response [1]. It is considered to be endemic in about 98 countries worldwide including India, Bangladesh, Brazil, Ethiopia and Sudan consisting about more than $90 \%$ of total cases [2].

Leishmaniasis is considered to be disease of lowland as climate and geographical factors play an important role in the distribution of vector, parasite and reservoir [3, 4]. However, recently few studies have reported leishmaniasis at higher altitude of Himalayan and sub

Manuscript received: $28^{\text {th }}$ May 2019

Reviewed: $4^{\text {th }}$ June 2019

Author Corrected: $10^{\text {th }}$ June 2019

Accepted for Publication: $15^{\text {th }}$ June 2019
Himalayan region of India [5, 6, 7]. LD bodies, the amastigote forms of the parasite which are demonstrated in the bone marrow are associated with various morphological changes which may give clue to the diagnosis of the infection. Literature search shows that studies have been conducted to study the bone marrow examination in leishmaniasis both in endemic and non endemic regions [5, 8,9]. These studies have classified the associated morphological features on bone marrow into common, uncommon and atypical features which may help in diagnosing leishmaniasis.

The present study was also therefore conducted to study the clinico-hematological profile and the various morphological features on bone marrow examination associated with leishmaniasis in the non-endemic sub Himalayan region of India. 


\section{Material and Methods}

Setting and type of study: The prospective study was conducted in the Department of Pathology and Lab Medicine of the institute situated in the north Himalayan Uttarakhand state of India.

Sampling method: It included all the cases of leishmaniasis which were diagnosed on bone marrow examination over a period of one and half year from July 2017 till December 2019.

Inclusion criteria: Cases on bone marrow aspirate examination showing presence of LD bodies.

Exclusion criteria: Bone marrows aspirate examination which did not show any LD body.

\section{Results}

The study included total 10 cases of visceral leishmaniasis which were diagnosed on bone marrow aspirate cytology over

Table 1: Clinical presentation in cases showing LD bodies on bone marrow aspirate.

\begin{tabular}{|c|c|}
\hline Clinical Presentation & Number of cases \\
\hline Fever & 10 \\
\hline Hepato-splenomegaly & 07 \\
\hline Nausea and Vomiting & 06 \\
\hline Lymphadenopathy & 02 \\
\hline Loss of weight/appetite & 05 \\
\hline Jaundice & 01 \\
\hline
\end{tabular}

Table 2 : Hematological and Bone marrow findings in Leishmaniasis

\begin{tabular}{|l|c|c|}
\hline Peripheral Blood Examination & Number of cases & Percentage \\
\hline Anemia & 09 & 90 \\
\hline Pancytopenia & 09 & 80 \\
\hline Leukopenia & 08 & 80 \\
\hline Thrombocytopenia & 08 & 60 \\
\hline Nucleated red blood cell & 06 & 20 \\
\hline Monocytosis & 02 & 100 \\
\hline Bone marrow aspirate cytology & & 80 \\
\hline Plasmacytosis & 10 & 30 \\
\hline Increased histiocytes & 08 & 20 \\
\hline Intra histiocytic LD bodies & 03 & 90 \\
\hline Extra histiocytic LD bodies & 02 & 70 \\
\hline Mixed Intra and Extrahistiocytic LD bodies & 09 & 30 \\
\hline Hemophagocytosis & 07 & 70 \\
\hline Dysmyelopoiesis & 03 & \\
\hline Erythroid hyperplasia & 07 & \\
\hline
\end{tabular}




\section{Original Research Article}

a period of one and half year. All the cases were resident of Uttarakhand, a non-endemic hilly state of India and there was also no history of visit to any endemic area. Out of total cases, 6 cases were resident of places which are at the height of 800 meter or above sea level. Table 1 shows the clinico-hematological presentation of the cases. It shows that fever and hepato-splenomegaly was the most common clinical presentation with pancytopenia ( 9 cases) and anemia ( 8 cases) being the most common hematological findings. 8 cases showed low parasite density score of 1-2+. Table 2 shows the associated morphological features observed on bone marrow aspirate cytology in cases demonstrating LD bodies. It showed that plasmacytosis ( 9 cases) and hemophagocytosis $(8$ cases) were the most common features (Figure 1). It was also observed that LD bodies were present both extra cellular and intra cellular (Figure 2,3) One case was clinically suspected as acute leukemia which turned out to be a case of leishmaniasis on bone marrow examination. RK 39 antigen detection test was done only in three cases and was positive in all of them.

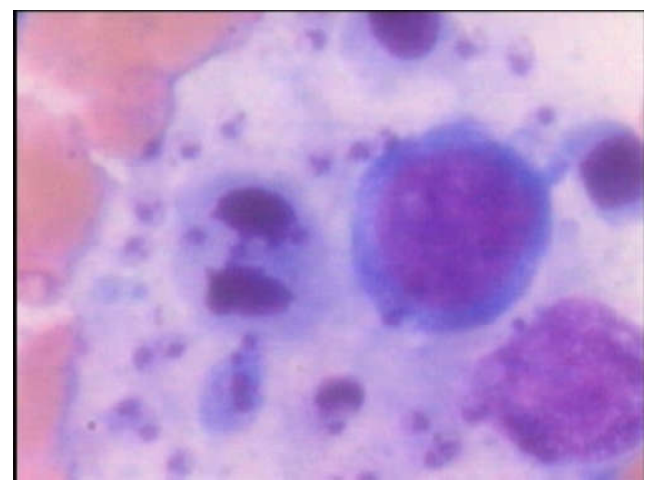

Figure-1: Bone marrow aspirate cytology showing hemophagocytosis with LD bodies (May Grunwald Giiemsa, x100)

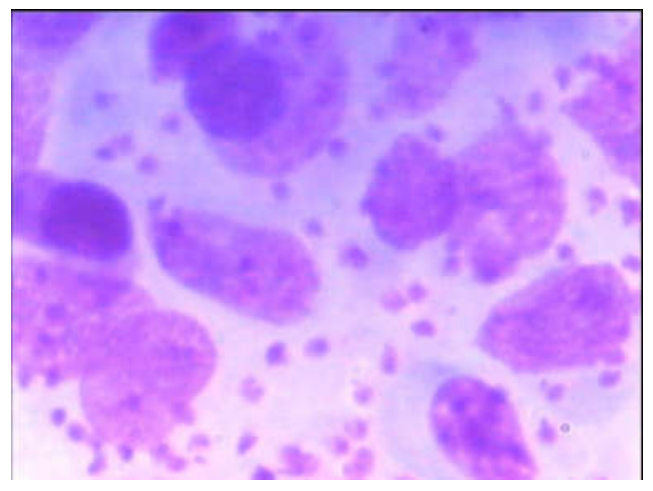

Figure-2: Bone marrow aspirate cytology showing intracellular LD bodies (May GrunwaldGiiemsa, x100)

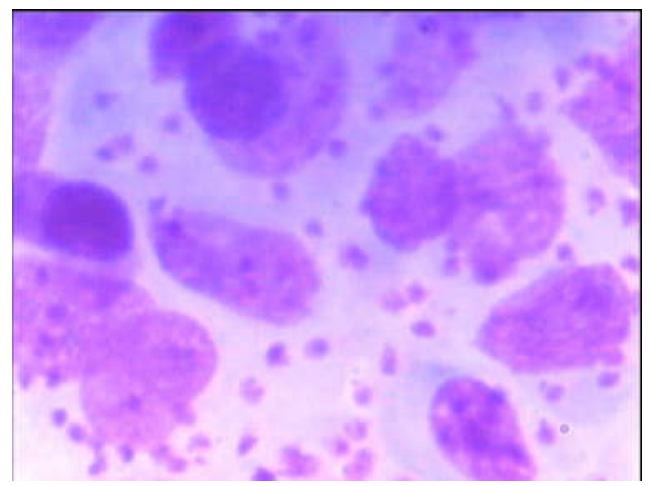

Figure-3: Bone marrow aspirate cytology showing extracellular LD bodies (May GrunwaldGiiemsa, x100)

\section{Discussion}

India accounts for major burden of leishmaniasis worldwide with approximately 146,700-282,800 new cases being reported each year [10]. Although the disease is common in Bihar, West Bengal and other eastern states of India but a new focus of leishmaniasis is being reported in Himalayan and sub Himalayan regions of India in few recent studies [7, 11, 12]. Present study also reported new 10 cases of leishmaniasis over period of one and half year in our non-endemic sub-Himalayan region. The probable reason of this new focus may be associated with change in climatic condition, deforestation which may have resulting in change of vector breeding. In addition, increased migration of population from endemic regions may have also resulted in new cases of leishmaniasis in this hilly region. The main reason of population migration may be due to high rate of pilgrimage in this state along with major hydroelectric projects which also attract population for employment from other states of India. In the present study fever and splenomegaly was the most common presentation and disease was mostly clinically unsuspected in most of the cases. The probable reason was because of its presence in nonendemic region and the clinical features mimic with 


\section{Original Research Article}

other common diseases like malaria, liver diseases or enteric fever. It was also observed that one case was suspected of acute leukemia which turned out to be of leishmaniasis on bone marrow examination. This itself lays the importance of the fact that even in nonendemic regions. leishmaniasis may be considered in differential diagnosis in the light of clinical features. However, fever and hepato-splenomegaly are the chief clinical presentation in other leishmaniasis studies also [13].

Pancytopenia was the most common presentation on peripheral blood examination in the present study. Although few studies from non-endemic regions have also reported pancytopenia as common presentation on peripheral blood examination but another study observed pancytopenia in only $10 \%$ of cases in leishmaniasis [8, 9, 14]. The authors suggest that pancytopenia as the most common presentation in the present study may be due to the fact that cases presented late to the tertiary care centre from remote hilly areas.

On bone marrow examination plasmacytosis was observed in all the cases of leishmaniasis in the present study. Another interesting feature that was observed in the present study on bone marrow examination was presence of increased histiocytes and hemophagocytosis. The authors therefore suggest that vigilant search for LD bodies should be made even in unsuspected cases if histiocytosis and hemophagocytosis is present on bone marrow examination, associated with plasmacytosis. This will help in clinching of early diagnosis of leishmaniasis even in unsuspected cases. Literature search shows that certain previous studies have classified the associated hematological and morphological bone marrow in cases of leishmaniasis as common, uncommon and atypical features $[5,8,9]$.

Although Chandra et al and Bhatia et al have also observed hemophagocytosis as common feature on bone marrow examination in leishmaniasis but another study from endemic region by Daneshbod et al have reported hemophagocytosis as an uncommon cytological finding in leishmaniasis [5, 8, 9]. Studies have also observed that LD bodies aggregates on bone marrow aspirate in form of rosettes, ring and strap shape which may be confused with fungal spores and therefore pathologists should be aware of such presentation to avoid misdiagnosis [5]. Interestingly, it has been previously reported that LD bodies may also be observed in non histiocytic cells like polymorphs, megakaryocytes and metamyelocyte [5]. Although, none of the case in the present study showed the presence of LD bodies in non histiocytic cells but the pathologist should also keep this feature in mind so as to avoid missing of diagnosis. In addition, the presence of cytoplasmic and granular bodies have also been demonstrated on bone marrow examination in certain studies from endemic region [9]. However this phenomenon was not observed in the present study which may be related to low parasitic density in our non endemic region.

It has been suggested previously that release of cysteine proteinase by LD bodies may be responsible for lysis of cytoplasm [9]. In addition to bone marrow aspirate examination, the trephine biopsy may also show certain changes in leishmaniasis. Granulomas, necrosis, increased vascularity are considered to be the features which may be observed on biopsy. The parasitic density plays an important role in pathogenesis of these features as high parasitic density in endemic regions may lead to more granuloma formation and necrosis.

However, in India an important differential diagnosis of tuberculosis should also be excluded by vigilant examination of bone marrow smears and biopsy. Ziehl Neelsen stain for the acid fast tubercle bacilli may be helpful in this regard. Singh et al have concluded that visualization of LD body using microscopy is confirmatory diagnostic test for leishmaniasis but which may not be possible in endemic areas. rK39 ELISA has emerged as an important laboratory test for diagnosis of leishmaniasis with high specificity and sensitivity [15].

\section{Conclusion}

Leishmaniasis is emerging as an important major parasitic infection in the non-endemic Himalayan and sub Himalayan region of India. Fever, splenomegaly and pancytopenia are important clinical and peripheral blood examination features associated with this disease. Hemophagocytosis and plasmacytosis on bone marrow examination should prompt for vigilant search for LD bodies in the marrow to avoid missing of diagnosis even in unsuspected cases.

\section{Authors' Contributions}

AK Gupta: Conception and design of study, data acquisition and analysis

H. Chandra: Design of study, data acquisition and analysis, interpretation of data and drafting of manuscript

V. Bharati: Collection of data, literature search

N. Singh: Collection of data, intellectual input

S. Kishore: Intellectual input, critical analysis 
Original Research Article

\section{What this study adds to existing knowledge?}

Leishmaniasis is emerging as an important parasitic infection in non-endemic Himalayan and sub Himalayan region of India. Hemophagocytosis and plasmacytosis on bone marrow examination should prompt for vigilant search for LD bodies in marrow to avoid missing of diagnosis even in unsuspected cases.

Findings: Nil; Conflict of Interest: None initiated Permission from IRB: Yes

\section{References}

1.Herwaldt BL."Leishmaniasis in Harrision's Principles of Internal Medicine. FauciAS,Braunwald E, Kasper DL, et al.Eds,pp1296-1300 McGraw Hill, New York, NY,USA, $17^{\text {th }}$ ed 2008.

2. Ponirovsky E N. Bi-regional meeting on leishmanias is to strengthen cross border collaboration for the control of leishmaniasis in Central Asian Countries (WHO regional office for Europe) and Middle East countries (WHO regional office for the Eastern Mediterranean) WHO and Turkmenistan. Med Parazitol 2015, 3 (3):62-63.

3. Bhunia GS, Kesari S, Jeyaram A, et al. Influence of topography on the endemicity of Kala-azar: a study based on remote sensing and geographical information system. Geospat Health. 2010;4(2):155-65. DOI:10. 4081 /gh.2010.197

4. Elnaiem DE, Schorscher J, Bendall A, Obsomer V, Osman ME, Mekkawi AM, et al. Risk mapping of visceral leishmaniasis: the role of local variation in rainfall and altitude on the presence and incidence of kala-azar in eastern Sudan. Am J Trop Med Hyg. 2003;68(1):10-7.

5. Chandra H, Chandra S, Kaushik RM. Visceral leishmaniasis with associated common, uncommon, and atypical morphological features on bone marrow aspirate cytology in nonendemic region. J Trop Med. 2013;2013. DOI:https://doi.org/10.1155/2013/861032

6. Mahajan SK, Machhan P, Kanga A, Thakur S, Sharma A, Prasher BS, et al. Kala-Azar at high altitude. J Commun Dis. 2004 Jun;36(2):117-20.
7. Kumar A, Vinita R, Thapliyal N, Saxena SR. Kalaazar--a case series from non endemic area, Uttarakhand. J Commun Dis. 2012;44(3):145-9.

8. Bhatia P, Haldar D, Varma N, Marwaha RK, Varma $\mathrm{S}$. A case series highlighting the relative frequencies of the common, uncommon and atypical/unusual hematological findings on bone marrow examination in cases of visceral leishmaniasis. Mediter J Hematol Infect Dis. 2011;3(1):e2011035. DOI: 10.4084/ MJHID. 2011.035

9. Daneshbod Y, Dehghani SJ, Daneshbod K. Bone marrow aspiration findings in kala-azar. Acta Cytol. 2010;54(1):12-24. DOI:10.1159/000324961

10. Alvar J, Vélez ID, Bern C, Herrero M, Desjeux P, Cano J. Leishmaniasis worldwide and global estimates of its incidence. PLoS One. 2012;7(5):e35671. DOI: 10.1371/journal.pone.0035671. Epub 2012 May 31.

11. Ahmad S, Chandra H, Bhat NK, Dhar M, Shirazi N, Verma SK. North Indian state of Uttarakhand: a new hothouse of visceral leishmaniasis. Trop Doct. 2016;46 (2): 111-3. DOI: 10.1177/0049475515609245. Epub 2015 Oct 14 .

12. Joshi A, Gulati A, Pathak VP, Bansal R. Post-KalaAzar-dermal-leishmaniasis: an unusual presentation from Uttarachal (a non-endemic hilly region of India). Indian J Dermatol Venereol Leprol. 2002;68(3):171-3.

13. Prieto Tato LM, La Ordem Izquierdo E, Guillen Martin S, Salcedo Lobato E, Garcia Esteban C, Garcia Bermejo I, et al. Visceral childhood leishmaniasis: diagnosis and treatment. An Pediatr (Barc) 2010;72 (5):347-351. DOI:10.1016/j.anpedi.2009.12.020

14. Varma N, Naseem S. Hematologic Changes in Visceral Leishmaniasis/Kala Azar. Indian J Hematol Blood Transfus. 2010; 26(3):78-82. DOI: 10.1007/s 12288-010-0027-1.

15. Singh OP, Sundar S. Developments in diagnosis of visceral leishmaniasis in the elimination era. Journal of parsasitology research. 2015;2015. DOI: https://doi. org / 10. 1155/2015/239469.

\section{How to cite this article?}

Gupta A.K, Chandra H, Bharati V, Singh N, Kishore S. Clinico-hematological profile and morphological features on bone marrow aspirate in visceral leishmaniasis from non-endemic region. Trop J Path Micro 2019;5(6):408-412.doi:10. 17511/jopm.2019.i06.12. 